AS-TEXONO/04-03

September 4, 2018

\title{
Neutrino Magnetic Moments: Status and Prospects
}

\author{
Henry T. Wong ${ }^{\mathrm{a}}$ \\ ${ }^{a}$ Institute of Physics, Academia Sinica, Taipei 11529, Taiwan.
}

Finite neutrino magnetic moments are consequences of non-zero neutrino masses. The particle physics foundations of the subject are summarized. The astrophysical bounds as well as the results from recent direct experiments are reviewed. Future projects and prospects are surveyed.

\section{INTRODUCTION}

The strong evidence of neutrino oscillations from the solar, atmospheric and long baseline accelerator and reactor neutrino measurements implies finite neutrino masses and mixings 12. Their physical origin and experimental consequences are not fully understood. Experimental studies on the neutrino properties and interactions can shed light to these fundamental questions and provide constraints to the interpretations in the future precision oscillation experiments. New and improved neutrino sources and detector technologies have to be developed in parallel for such studies.

The couplings of neutrinos with the photons are generic consequences of finite neutrino masses, and are one of the important intrinsic neutrino properties 3 to explore. The neutrino electromagnetic vertex can be parametrized by terms with $\gamma_{\eta}$ and $\sigma_{\eta \xi}$ corresponding to interactions without and with its spin, respectively identified as the "neutrino charge radius" and "neutrino magnetic moments", the latter of which is the subject of this review ${ }^{1}$.

\section{PARTICLE PHYSICS OVERVIEW}

The most general form for the effective Lagrangian describing the spin component of the neutrino electromagnetic vertex can be expressed

\footnotetext{
${ }^{1}$ Proceedings to the XXIst International Conference on Neutrino Physics and Astrophysics, Paris, 2004
}

as

$L=\frac{1}{2} \bar{\nu}_{j} \sigma_{\eta \xi}\left(\beta_{i j}+\epsilon_{i j} \gamma_{5}\right) \nu_{i} F^{\eta \xi}+h . c$.

where $\epsilon_{i j}$ and $\beta_{i j}$ are respectively the electric and magnetic dipole moments which couple together the neutrino mass eigenstates $\left(\nu_{i}\right)_{L}$ and $\left(\nu_{j}\right)_{R}$, resulting in a change of the spin-state. Cases where $\nu_{i}=\nu_{j}$ and $\nu_{i} \neq \nu_{j}$ correspond to diagonal and transitional moments, respectively. Symmetry principles as well as neutrino properties place constraints to the matrices $\epsilon_{i j}$ and $\beta_{i j}[4]$. For example, Majorana neutrinos require $\epsilon_{i i}=\beta_{i i}=0$ which implies the diagonal moments vanishes. The study of neutrino electromagnetic properties is, therefore, in principle a way to distinguish between Dirac and Majorana neutrinos.

The experimental observable "neutrino magnetic moment" $\left(\mu_{\nu}\right)$, usually expressed in units of the Bohr magneton $\left(\mu_{\mathrm{B}}\right)$, for neutrinos with energy $E_{\nu}$ produced as $\nu_{l}$ at the source and after traversing a distance $\mathrm{L}$ can be described by

$\mu_{\nu}^{2}\left(\nu_{l}, L, E_{\nu}\right)=\sum_{j}\left|\sum_{i} U_{l i} e^{-i E_{\nu} L} \mu_{i j}\right|^{2}$

where $\mu_{i j} \equiv\left|\beta_{i j}-\epsilon_{i j}\right|$ and $U_{l i}$ is the neutrino mixing matrix. The observable $\mu_{\nu}$ is therefore an effective and convoluted parameter and the interpretations of experimental results depend on the exact $\nu_{l}$ compositions at the detectors. Accordingly, the $\mu_{\nu}$ limits from reactor experiments are not identical to those from ${ }^{8} \mathrm{~B}$ solar neutrino experiments, which in turn are different to those from ${ }^{7} \mathrm{Be}$ solar neutrino experiments. 
Given a specific model, $\mu_{\nu}$ can be calculated from first principles. Minimally-Extended Standard Model with massive Dirac neutrinos 1 gives $\mu_{\nu} \sim 10^{-19}\left[m_{\nu} / 1 \mathrm{eV}\right]$ which is far too small to have any observable consequences. Incorporation of additional physics, such as Majorana neutrino transition moments or right-handed weak currents, can significantly enhance $\mu_{\nu}$ to the experimentally relevant ranges 115. Supersymmetry can also contribute to the process, and the consequences from models based on extra-dimensions were recently discussed $[\underline{\underline{6}}$.

Information on $\mu_{\nu}$ can be derived from astrophysics arguments as well as from direct laboratory experiments. The various manifestations of the neutrino-photon couples are shown Figure 1 In particular, studies of neutrino-electron scatterings are the most robust and established methods. A finite $\mu_{\nu}$ gives rise to an additional contribution in the $\nu$-e scattering differential cross-section [5]

$\left(\frac{d \sigma}{d T}\right)_{\mu_{\nu}}=\frac{\pi \alpha_{e m}^{2}}{m_{e}^{2}}\left[\frac{1-T / E_{\nu}}{T}\right] \mu_{\nu}^{2}$

where $T$ the electron recoil energy, the experimental measurable. The neutrino radiative decay rate $\Gamma_{i j}$ for the process $\nu_{i} \rightarrow \nu_{j}+\gamma$ is related to $\mu_{i j}$ via[ 7 ]

$\Gamma_{i j}=\frac{1}{8 \pi} \frac{\left(m_{i}^{2}-m_{j}^{2}\right)^{3}}{m_{i}^{3}} \mu_{i j}^{2}$,

where $m_{i, j}$ are the masses for neutrino masseigenstates $\nu_{i, j}$.

\section{ASTROPHYSICS BOUNDS}

Astrophysics bounds on $\mu_{\nu}$ were mostly derived from the consequences from a change of the neutrino spin-states in the astrophysical medium 1. 8. These include studies in the available degrees of freedom in Big Bang Nucleosynthesis, stellar cooling via plasmon decay, and the cooling of supernova 1987a. The typical range is $\mu_{\nu}($ astro $)<10^{-10}-10^{-12} \mu_{\mathrm{B}}$.

The bounds, however, depends on modeling of the astrophysical systems, as well as on placing certain assumptions on the neutrino properties. The supernovae cooling arguments only apply for Dirac neutrinos where the right-handed
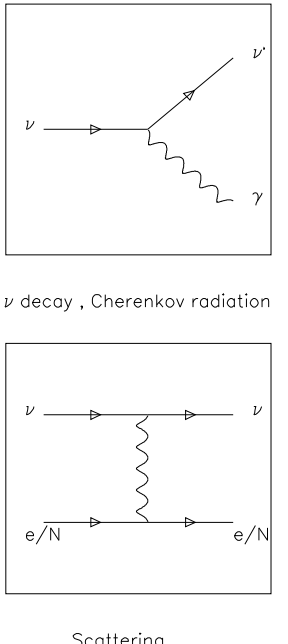

Scattering $\nu$ decay, Cherenkov radiation

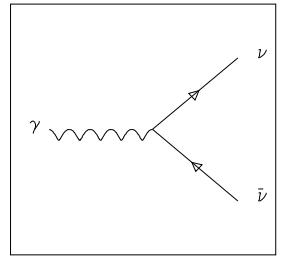

$\gamma$ decay (plasma)

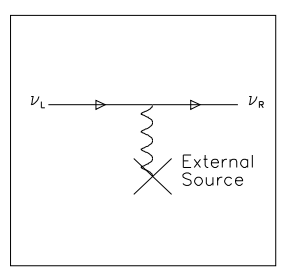

Spin precession
Figure 1. Schematic diagrams for the various experimental manifestations of neutrino-photon couplings.

state is sterile and can leave the astrophysical objects readily. Another generic assumption is the absence of other non-standard neutrino interactions except for an anomalous magnetic moment. For more realistic studies, a global treatment would be desirable, incorporating oscillation effects, matter effects as well as the complications due to interference and competitions among various channels.

As a historical footnote, the spin-flavor precession (SFP) mechanism, with or without matter resonance effects in the solar medium, has been used to explain solar neutrino deficit 9 . The solar $\nu_{e}$ would interact with solar magnetic field $\mathrm{B}_{\odot}$ via its magnetic moment to become $\nu_{x}(x \neq e)$. This scenario is in fact compatible with all solar neutrino data. The terrestrial KamLAND experiment, however, recently confirmed the Large Mixing Angle (LMA) parameter space of the matter oscillation scenario as the solution for the solar neutrino problem[12], such that SFP can be excluded as the dominant contribution in solar neutrino physics. Conversely, coupling the LMA allowed region with the recent KamLAND solar- $\overline{\nu_{e}}$ 
bounds of $\overline{\nu_{e}} / \nu_{\odot}<2.8 \times 10^{-4}[10$, a constraint on $\int \mu_{\nu}\left[\mathrm{B}_{\odot} \perp\right] \mathrm{dr}$ can be derived, where $\mathrm{B}_{\odot \perp}$ denotes transverse component of the solar magnetic field. Recent work on the modeling of $\mathrm{B}_{\odot}[11$ turned this into bounds on the magnetic moments, also in the range of $\mu_{\nu}\left({ }^{8} \mathrm{~B}-\nu_{\odot}\right)<10^{-10}-10^{-12} \mu_{\mathrm{B}}$.

\section{RECENT RESULTS FROM DIRECT EXPERIMENTS}

Direct laboratory experiments on neutrino magnetic moments utilize solar, accelerator and reactor neutrinos as sources, and are conducted under controlled conditions. These approaches are robust and stay away from the ambiguities and model-dependence in the astrophysical bounds. The experiments require an understanding of the neutrino energy spectrum as well as its flavor/mass-eigenstate compositions at the detectors by independent means. They typically study neutrino-electron scatterings $\nu_{l}+e \rightarrow \nu_{x}+e$. The signature is an excess of events over those due to Standard Model (SM) and other background processes, which exhibit the characteristic $1 / \mathrm{T}$ spectral dependence. Limits from negative searches are valid for both Dirac and Majorana neutrinos and for all final states $\nu_{x}$, that is, for both diagonal and transitional moments. However, comparisons and interpretations among various experiments should take into account the difference in the compositions between them at the detectors.

\subsection{Solar Neutrinos}

Data from the solar neutrino and KamLAND experiments firmly established the validity of the Standard Solar Model (SSM) predictions of the solar neutrino flux, as well as the LMA-matter oscillation solution being the leading mechanism of neutrino flavor conversion in the Sun. This can be used as the basis of magnetic moment searches with solar neutrino data.

The Super-Kamiokande (SK) Collaboration performed spectral distortion analysis of their electron recoil spectral due to ${ }^{8} \mathrm{~B}$ solar neutrinoelectron scattering 12 . The study was to look for $1 / \mathrm{T}$ excess over an oscillation "background" at the 5-14 MeV energy range. SK data alone allowed a large region of $\left(\Delta m^{2}, \tan ^{2} \theta\right)$ param- eter space and could only set limit of $\mu_{\nu}\left({ }^{8} \mathrm{~B}\right.$ $\left.\nu_{\odot}\right)<3.6 \times 10^{-10} \mu_{\mathrm{B}}$ at $90 \%$ Confidence Level (CL). Coupling with constraints from the other solar neutrino and KamLAND results, the LMA region is uniquely selected as the solution, such that a more stringent limit of $\mu_{\nu}\left({ }^{8} \mathrm{~B}-\nu_{\odot ; L M A}\right)<$ $1.1 \times 10^{-10} \mu_{\mathrm{B}}$ at $90 \% \mathrm{CL}$ was derived.

The Borexino Collaboration performed analysis of their Counting Test Facility data at the ${ }^{7} \mathrm{Be}$ solar neutrino relevant range: $200-500 \mathrm{keV} 13$. Subtracting the known ${ }^{14} \mathrm{C} \beta$-spectrum and assuming an additional linear background, a fit to look for an $1 / \mathrm{T}$ spectrum did not indicate any excess and a limit of $\mu_{\nu}\left({ }^{7} \mathrm{Be}-\nu_{\odot}\right)<5.5 \times 10^{-10} \mu_{\mathrm{B}}$ at $90 \% \mathrm{CL}$ was derived, using $\mathrm{SSM}{ }^{7} \mathrm{Be} \nu_{\odot}$ flux.

An innovative insight is that neutrino magnetic moments can induce photo-dissociation in deuterium. The agreement between SNO neutralcurrent measurements with SSM $\nu_{\odot}$-flux predictions placed constraints to the $\nu_{e}-d$ neutralcurrents cross-sections and thus to the magnetic moment effects: $\mu_{\nu}\left({ }^{8} \mathrm{~B}-\nu_{\odot}\right)<3.7 \times 10^{-9} \mu_{\mathrm{B}}$ at 95\% CL 14 .

\subsection{Accelerator Neutrinos}

Accelerators provide neutrinos with known flavor compositions. The timing structures can be used for background subtraction. Compared to reactor neutrinos, the lower flux as well as higher energy limit the sensitivities. However, neutrinos of all three flavors are produced at accelerators such that this is the only laboratory avenue for studying magnetic moments from $\nu_{\mu}$ and $\nu_{\tau}$.

The LSND experiment measured "single electron" events from a beam with known $\nu_{e}, \nu_{\mu}$ and $\overline{\nu_{\mu}}$ fluxes and spectral compositions [15]. Taking the SM calculated values of $\sigma\left(\nu_{\mu}-e\right)$ and $\sigma\left(\overline{\nu_{\mu}}-\right.$ $e)$ which were confirmed by other experiments, the value of $\sigma\left(\nu_{e}-e\right)$ was derived. It agreed well with SM predictions and provided a measurement of $\sin ^{2} \theta_{W}=0.248 \pm 0.051$. Limits of $\mu_{\nu}\left(\nu_{e}\right)<$ $1.1 \times 10^{-9} \mu_{\mathrm{B}}$ and $\mu_{\nu}\left(\nu_{\mu}\right)<6.8 \times 10^{-10} \mu_{\mathrm{B}}$ at $90 \%$ CL were derived from the absence of excess of counts.

The DONUT experiment first observed explicit $\nu_{\tau}$ charged-current interactions 1 showing that the $\nu_{\tau}$ flux at a beam dump configuration is consistent with the expected level. The experiment 
also looked for possible "single-electron" events at cross-sections much larger than SM expectations. One event was observed while the predicted background from other known sources was 2.3 [16]. This was used to convert into a magnetic moment limit for $\nu_{\tau}: \mu_{\nu}\left(\nu_{\tau}\right)<3.9 \times 10^{-7} \mu_{\mathrm{B}}$ at 90\% CL.

\subsection{Reactor Neutrinos}

Reactor neutrino experiments provide the most sensitive laboratory searches for the magnetic moments of $\overline{\nu_{e}}$, taking advantages of the high $\overline{\nu_{e}}$ flux, low $\mathrm{E}_{\nu}$ and better experimental control via the reactor ON/OFF comparison. Neutrino-electron scatterings were first observed in the pioneering experiment 17] at Savannah River. A revised analysis of the data by Ref [5] with improved input parameters gave a positive signature consistent with the interpretation of a finite $\mu_{\nu}$ at $\mu_{\nu}\left(\overline{\nu_{e}}\right) \sim 2-4 \times 10^{-10} \mu_{\mathrm{B}}$.

The MUNU experiment [18] at the Bugey reactor in France deployed a Time Projection Chamber (TPC) filled with $\mathrm{CF}_{4}$ gas at 3 bar having a mass of $11.4 \mathrm{~kg}$, surrounded by active liquid scintillator as anti-Compton vetos. It gave excellent single-electron event selection and measured the scattering angle with respect to the reactor core direction. Neutrino events are scattered "forward" such that a forward/backward comparison was used to subtract background. The residual spectra from 66.6 days of reactor ON data are depicted in Figure 2 The residual counts above $\mathrm{T}=900 \mathrm{keV}$ were consistent with SM expectations, while an excess of events at $300 \mathrm{keV}<\mathrm{T}<900 \mathrm{keV}$ was observed where the origins remain unknown. Various limits were evaluated depending on the analysis threshold: $\mu_{\nu}\left(\overline{\nu_{e}}\right)<1.7 / 1.4 / 1.0 \times 10^{-10} \mu_{\mathrm{B}}$ at $90 \% \mathrm{CL}$ at $300,700,900 \mathrm{keV}$ threshold, respectively. The low energy $(<2 \mathrm{MeV})$ reactor neutrino spectra are not well-modeled 19 such that the possibility of yet-unaccounted-for neutrino production channels at the $\mathrm{MeV}$ energy range should be examined.

The TEXONO Collaboration adopted a compact all-solid design with an ultra-lowbackground high-purity germanium (ULB$\mathrm{HPGe}$ ) detector with a mass of $1.06 \mathrm{~kg}$ as target,
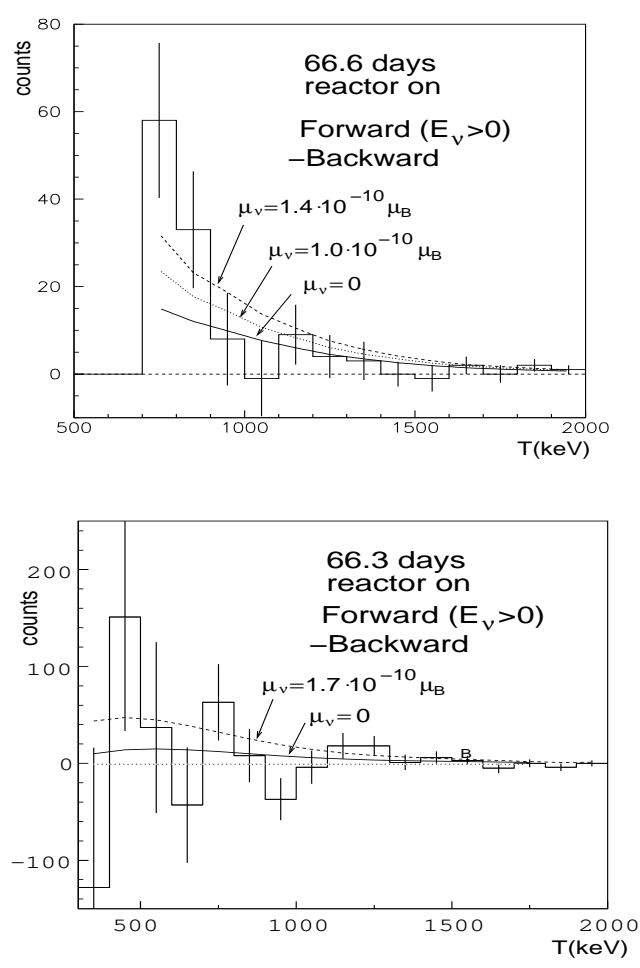

Figure 2. Residual spectra from MUNU for reactor-ON forward minus background events: visual scan $(>700 \mathrm{keV})$ and automatic scan $(>300$ $\mathrm{keV})$.

surrounded by anti-Compton detectors of $\mathrm{NaI}(\mathrm{Tl})$ and $\mathrm{CsI}(\mathrm{Tl})$ crystal scintillators, radon shields, passive shielding materials, and cosmic-ray veto with plastic scintillator panels. The measurement was performed at the Kuo-Sheng (KS) Power Plant in Taiwan.

The focus was on the $\mathrm{T}=10-100 \mathrm{keV}$ range for the enhanced signal rates and robustness in the control of systematic uncertainties. At this energy range, the $\nu$-e scattering rates due to magnetic moments are much larger than the SM rates at the $10^{-10} \mu_{\mathrm{B}}$ sensitivity level being explored, so that uncertainties in the irreducible SM background can be neglected [19]. In addition, $T \ll E_{\nu}$ such that the scattering rates due to $\mu_{\nu}$ depend 
on the total neutrino flux $\left(\phi_{\nu}\right)$ rather than the poorly-known details of the low-energy reactor neutrino spectra. The total neutrino flux is wellknown and can be evaluated accurately from reactor operation data - every fission is expected to produce about 6 and $1.2 \overline{\nu_{e}}$ 's due to $\beta$-decays of the fission daughters and of ${ }^{239} \mathrm{U}$ following neutron capture on ${ }^{238} \mathrm{U}$, respectively.

Comparing 4712/1250 hours of reactor ON/OFF data, no excess of events was found and with an analysis threshold of $12 \mathrm{keV}$ just above the complications due to atomic effects, a limit of $\mu_{\nu}\left(\overline{\nu_{e}}\right)<1.3 \times 10^{-10} \mu_{\mathrm{B}}$ at $90 \%$ CL was derived. The $\mathrm{ON} / \mathrm{OFF}$ and residual spectra are displayed in Figure [3 Another notable result is that a background level of $\sim 1 \mathrm{~kg}^{-1} \mathrm{keV}^{-1} \mathrm{day}^{-1}$ at the 10-20 keV range was achieved - a comparable range to those from the underground Dark Matter experiments.

Depicted in Figure $4 \mathrm{a}$ is the summary of the results in $\mu_{\nu}\left(\overline{\nu_{\mathrm{e}}}\right)$ searches versus the achieved threshold in reactor experiments. The dotted lines denote the $\mathrm{R}=\sigma(\mu) / \sigma(\mathrm{SM})$ ratio at a particular $\left[\mathrm{T}, \mu_{\nu}\left(\overline{\nu_{\mathrm{e}}}\right)\right]$. The large $\mathrm{R}$-values for the KS experiment imply that its results are robust against the uncertainties in the SM cross-sections. In particular, in the case where the excess events in Refs. 17 and 18 are due to unaccounted sources of neutrinos, the limits remain valid. Indirect bounds on the neutrino radiative decay lifetimes are inferred and displayed in Figure 40 for the simplified scenario where a single channel dominates the transition. It corresponds to $\tau_{\nu} \mathrm{m}_{\nu}^{3}>9.5 \times 10^{18} \mathrm{eV}^{3} \mathrm{~s}$ at $90 \% \mathrm{CL}$ in the nondegenerate case. Superimposed are the limits 20. from the previous direct searches of excess $\gamma$ 's from reactor and supernova SN1987a neutrinos, as well as the sensitivities of proposed simulated conversion experiments at accelerators. It can be seen that $\nu$-e scatterings give much more stringent bounds than the direct approaches.

\subsection{Global Analysis}

A global analysis was performed 21] fitting simultaneously the magnetic moment data from the reactor and solar neutrino experiments, and the LMA oscillation parameters constrained by solar neutrino and KamLAND results. Only Ma-
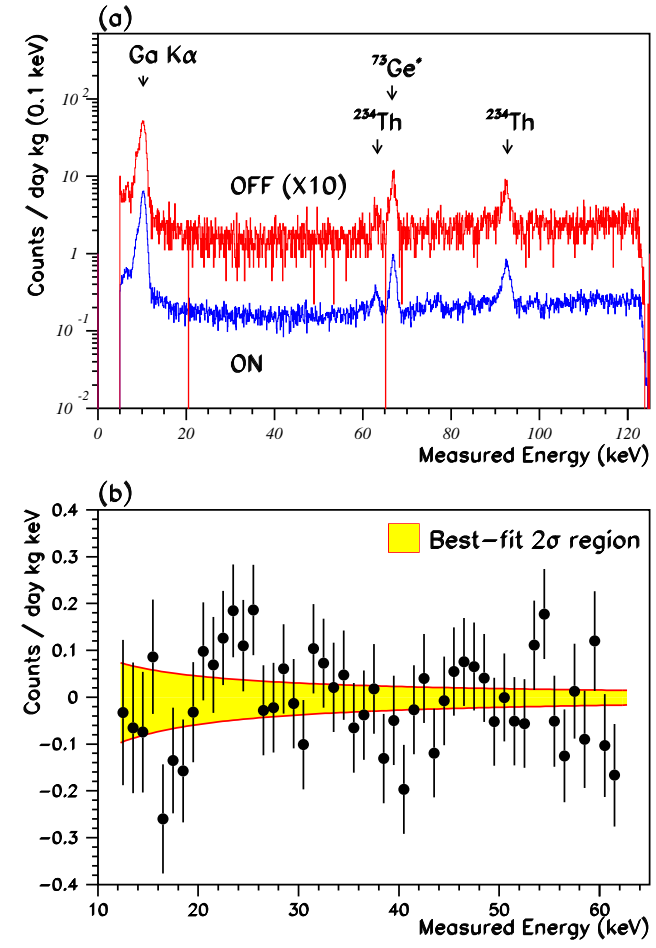

Figure 3. Data from TEXONO/KS on (a) reactor $\mathrm{ON} / \mathrm{OFF}$ energy spectra and (b) residual spectrum.

jorana neutrinos were considered such that there were only transition moments. A "total" magnetic moment vector $\Lambda=\left(\mu_{23}, \mu_{31}, \mu_{12}\right)$ was defined, such that its amplitude was given by $|\Lambda|^{2}=$ $\frac{1}{2} \operatorname{Tr}\left(\mu^{+} \mu\right)$. A global fit produced 90\% CL limits of $|\Lambda|<4.0 \times 10^{-10} \mu_{\mathrm{B}}$ from solar and KamLAND data, and $|\Lambda|<1.8 \times 10^{-10} \mu_{\mathrm{B}}$ when reactor data were added. The results indicate the role of reactor experiments in constraining the magnetic moment effects. 

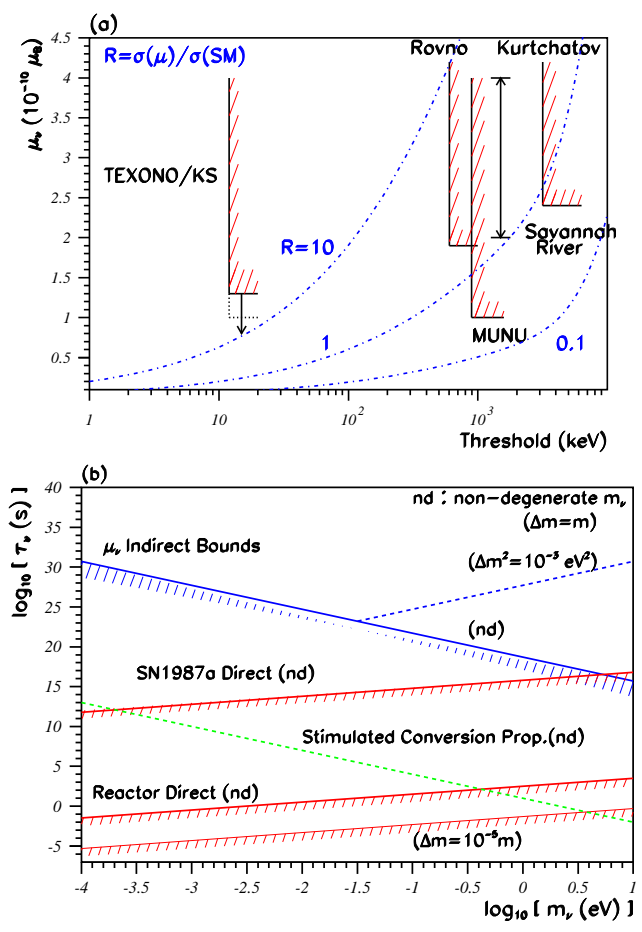

Figure 4. Summary of the results in (a) the searches of neutrino magnetic moments with reactor neutrinos, and (b) the bounds of neutrino radiative decay lifetime.

\section{FUTURE PROJECTS PROSPECTS}

AND

The sensitivities for neutrino magnetic moments in direct search experiments scale as

$\mu_{\nu} \propto \frac{1}{\sqrt{N_{\nu}}}\left[\frac{B}{M t}\right]^{\frac{1}{4}}$

where $N_{\nu}$ is the signal events at some reference magnetic moments, $B, M, t$ are the background level, detector mass and measurement time, respectively. It can be seen that the best strategy to improve on the sensitivities is to increase on $N_{\nu}$, which is proportional to the neutrino flux $\phi_{\nu}$ and is related to the detection threshold in recoil energy $\mathrm{T}$.
The atomic energy level effects 22 limit the potential enhancement of the sensitivities by reducing T only. For example, $N_{\nu}$ only increases by a factor of three with a lowering of detection threshold from $10 \mathrm{keV}$ to $10 \mathrm{eV}$ in Ge. Therefore, big statistical boost in $\mu_{\nu}$ will most favorably be achieved by an enhancement in $\phi_{\nu}$ - while keeping the systematics in control via (a) lowering the detection threshold to retain the " $\mu_{\nu} \gg S M$ " event-rate requirements, and (b) maintaining a low background level. Since the minimal energy transfer to the atomic electrons would be $\sim 100 \mathrm{eV}$, it follows from condition (a) that such an approach of enhancing $\phi_{\nu}$ and reducing $T$ may only be applicable down to a $\mu_{\nu}$ sensitivity range of $10^{-13} \mu_{\mathrm{B}}$.

The GEMMA experiment 23 under preparation at the Kalininskaya Nuclear Reactor in Russia is similar to the TEXONO-KS approach, aiming at an improvement to $\mu_{\nu}\left(\overline{\nu_{e}}\right) \rightarrow 3 \times 10^{-11} \mu_{\mathrm{B}}$ by locating at a closer distance, using a larger mass target and operating at a lower threshold. The MAMMONT project 24, currently at the R\&D phase, has ambitious specifications of deploying a $40 \mathrm{MCi}(4 \mathrm{~kg})$ tritium source with a flux of $6 \times 10^{14} \mathrm{~cm}^{-2} \mathrm{~s}^{-1}$ on ultra-sensitive detectors with threshold down to $10 \mathrm{eV}$, either with cryogenic silicon detectors or germanium with internal amplification. The projected sensitivity is $\mu_{\nu}\left(\overline{\nu_{e}}\right) \rightarrow 2.5 \times 10^{-12} \mu_{\mathrm{B}}$.

The TEXONO Collaboration continued data taking with the ULE-HPGe at KS. Sensitivities to the $\sim 10^{-10} \mu_{\mathrm{B}}$ range can be expected. In parallel, a $\mathrm{CsI}(\mathrm{Tl})$ crystal scintillator array [25] with a total mass of $200 \mathrm{~kg}$ is also collecting data. The strategy is to focus on the high $(>3 \mathrm{MeV})$ recoil energy range to perform a first measurement of SM neutrino-electron scattering at the $\mathrm{MeV}$ momentum transfer range. A prototype "Ultra-Low-Energy" germanium detector with an active mass of $5 \mathrm{~g}$ is being tested, with the goal of developing into a $1 \mathrm{~kg}$ Ge-array detector for the first experimental observation of neutrinonucleus coherent scattering. As by-product, such an experiment will potentially probe $\mu_{\nu}\left(\overline{\nu_{e}}\right) \rightarrow$ $2 \times 10^{-11} \mu_{\mathrm{B}}$. An energy threshold of $100 \mathrm{eV}$ has been demonstrated [26] with the prototype while background studies at the sub-keV range are un- 
der way at KS.

Alternatives of neutrino sources such as artificial radioactive sources 27 for $\mathrm{NaI}(\mathrm{Tl})$ and the Borexino detectors, as well as acceleratorbased $\beta$-sources for large TPCs 28, have been discussed, projecting a sensitivity range of $\sim$ few $\times 10^{-11} \mu_{\mathrm{B}}$ in both cases.

\section{OUTLOOK}

The magnetic moments of the neutrino parametrize how it couples to the photons and are sensitive to its masses and mixings, as well as its Dirac or Majorana nature. It is, therefore, a conceptually rich subject with much neutrino physics and astrophysics to be explored. However, there are no indications of any measurable/observable positive signatures in the current and future rounds of experimental efforts. Improvement in sensitivities will necessarily involve new neutrino sources as well as novel neutrino detection techniques and channels. These advances may find important potential applications in other areas of neutrino and underground physics experimentations.

\section{REFERENCES}

1. See the respective sections in Review of Particle Physics, Particle Data Group, Phys. Lett. B 592 (2004), for details and references.

2. See these proceedings for updates.

3. A. de Gouvea, these proceedings.

4. B. Kayser, Phys. Rev. D 26, 1662 (1982); J.F. Nieves, Phys. Rev. D 26, 3152 (1982).

5. P. Vogel and J. Engel, Phys. Rev. D 39, 3378 (1989), and references therein.

6. R.N. Mohapatra, S.P. Ng, H.B. Yu, hep-ph/0404274 (2004).

7. G.G. Raffelt, Phys. Rev. D 39, 2066 (1989).

8. G.G. Raffelt, "Stars as Laboratories for Fundamental Physics", Sect. 7.5, U. Chicago Press (1996).

9. J. Barranco et al., Phys. Rev. D 66, 093009 (2002), and references therein.

10. K. Eguchi et al., Phys. Rev. Lett. 92, 071301 (2004).

11. E.Kh. Akhmedov and J. Pulido, Phys. Lett.
553, 7 (2003); O.G. Miranda et al., Phys. Rev. Lett. 93, 051304 (2004).

12. D.W. Liu et al., Phys. Rev. Lett. 93 021802, (2004).

13. H.O. Back et al., Phys. Lett. B 563, 35 (2003).

14. J.A. Grifols, E. Masso and S. Mohanty, Phys. Lett. B 587184 (2004).

15. L.B. Auerbach et al., Phys. Rev. D 63, 112001 (2001).

16. R. Schwienhorst et al., Phys. Lett. B 513, 23 (2001).

17. F. Reines, H.S. Gurr and H.W. Sobel, Phys. Rev. Lett. 37, 315 (1976).

18. Z. Daraktchieva et al., Phys. Lett. B 564, 190 (2003).

19. H.B. Li and H.T. Wong, J. Phys. G 28, 1453 (2002).

20. H.B. Li et al., Phys. Rev. Lett. 90, 131802 (2003), and references therein.

21. W. Grimus et al., Nucl. Phys. B 648, 376 (2003); M.A. Tortola, hep-ph/0401135 (2004).

22. V.I. Kopeikin et al., Phys. Atom. Nucl. 60, 2032 (1997).

23. A.G. Beda et al., Phys. Atom. Nucl. 61, 66 (1998).

24. L.N. Bogdanova, Nucl. Phys. A 721499 (2003)

25. H.T. Wong et al., Astropart. Phys. 14, 141 (2000); H.B. Li et al., Nucl. Instrum. Methods A 459, 93 (2001); Y. Liu et al., Nucl. Instrum. Methods A 482, 125 (2002).

26. H.T. Wong, Mod. Phys. Lett. A 19, 1207 (2004).

27. I.R. Barabanov et al., Astropart. Phys. 8, 67 (1997); A. Ianni and D. Montanino, Astropart. Phys. 10, 331 (1999).

28. G.C. McLaughlin and C. Volpe, Phys. Lett. B 591, 229 (2004); C. Volpe, these proceedings. 